\title{
PARADIGMEN UND TEXTSORTEN LEBENSGESCHICHTLICHEN ERZÄHLENS
}

Zu Beginn der kleinen Erzählung Die Amsel, veröffentlicht 1936 im Bändchen Nachlaß zu Lebzeiten, läßt Robert Musil seinen Ich-Erzähler über Jugendfreundschaft reflektieren:

Denn im Grunde, heißt es da, ist Jugendfreundschaft um so sonderbarer, je älter man wird. Man ändert sich im Laufe solcher Jahre vom Scheitel bis zur Sohle und von den Härchen der Haut bis ins Herz, aber das Verhältnis zueinander bleibt merkwürdigerweise das gleiche und ändert sich sowenig wie die Beziehungen, die jeder einzelne Mensch zu den verschiedenen Herren pflegt, die er der Reihe nach mit Ich anspricht. Es kommt ja nicht darauf an, ob man so empfindet wie der kleine Knabe mit dickem Kopf und blondem Haar, der einst photographiert worden ist; nein, man kann im Grunde nicht einmal sagen, daß man dieses kleine, alberne, ichige Scheusal gern hat. ${ }^{1}$

Was Musil ins einprägsame Bild des albernen, ichigen Scheusals verdichtet, das man einmal war und einem gänzlich abhanden gekommen ist, veranschaulicht die Einsicht von der diachronen Differenz im normalerweise integrierend vorgehenden Selbstverständnis des Subjekts und verweist auf dessen Artifizialität. Die über das Ende hinaus rätselhaft bleibende Geschichte des Azwei, die, typisch für lebensgeschichtliches Erzählen, stationenhaft dargeboten wird - sie hat $\mathrm{zu}$ vielerlei reizvollen, konträren Interpretationen Anlaß gegeben -, ist freilich eine fiktionale, wiewohl sie, Musils Tagebüchern nach zu schließen, durchaus autobiographische Erlebnisse gestaltet. Für ein Genre, das, wie eben das (auto)biographische, in erster Linie seine Faktizität hervorstreicht, mag jedoch Franz Grillparzers Selbstbiographie relevanter erscheinen. Sie reicht bis 1836, genau hundert Jahre bevor Musils kleiner Text erscheint; aufgefunden und veröffentlicht wurde sie erst nach Grillparzers Tod, die Niederschrift - im Auftrag der 1848 gegründeten Österreichischen Akademie - datiert von 1853. Diese Lebensbeschreibung begeisterte und inspirierte bereits Musil, Kraus und Kafka; wie Musils Amsel ist ihr von Lesern und von der Forschung in den letzten Jahren erneut große Aufmerksamkeit geschenkt worden. Zurecht, denn, ohne das Biographisieren explizit zu thematisieren, räsonniert Grillparzer darin wiederholt über die seltsamen Wege der Erinnerung - Langeweile, Bewunderung und Güte waren dafür in seinem Fall besonders 
mißlich - und läßt den retrospektiven Interpretationscharakter des Lebenslaufs durchscheinen. $^{2}$

\section{Das traditionelle lebensgeschichtliche Paradigma und seine narrative Struktur:}

Im Gegensatz zum deutlichen Bewußtsein vom Konstruktionscharakter lebensgeschichtlichen Erzählens, wie wir es bei hervorragenden Künstlern nachweisen können, setzt sich Anfang des 20. Jahrhunderts zunehmend ein Genreverständnis durch, das in hohem Maße sowohl Produktion, Textstruktur wie auch Rezeption bis in die Gegenwart bestimmt und den Wünschen und Forderungen breiter, durch die literarischen Entwicklungen sowie durch die allgemeine Wirklichkeitserfahrung verunsicherter Leserschichten entgegenkommt: das sogenannte traditionelle lebensgeschichtliche Paradigma.

Die Vorbehalte gegen dieses Paradigma sind kombiniert strukturell und ideologiekritisch. Vom deviationsästhetischen Standpunkt aus, einem Literaturverständis, das die Mündigkeit des Lesers und die Subversivität des Ästhetischen akzentuiert, gehört der Großteil der Biographik, m.E. der Autobiographik und vor allem der Memoirenliteratur mit seiner Flucht in die Vergangenheit aus mangelnder Fähigkeit zur Gegenwartsbewältigung, seinem Rückzug auf das Individuum, was sowohl eine Reprivatisierung der Geschichts- und Gesellschaftsdarstellung wie auch die Entpolitisierung des Einzelnen zur Folge hat, sowie mit seiner nostalgischen Rückwendung auf inexistente alte Zeiten deutlich der affirmativen Kultur ${ }^{3}$ an. Im Gegensatz zur Unverbindlichkeit fiktionaler Konsolationsliteratur kommen (auto)biographische Texte durch ihre zumindest antinomische (eigentlich triadische) Struktur, durch ihre vermeintliche Verankerung in der Realität und Pragmatik einerseits und ihren verhüllten Konstruktionsbzw. Fiktionscharakter andererseits breiten Kreisen von existentiell, sozial, altersbedingt, psychisch oder ästhetisch Desorientierten äußerst entgegen. Die Erfüllung konträrer Bedürfnisse kann sogar beträchtlich wirkungsvoller geleistet werden als mithilfe der Trivialliteratur, deren viel geringeres Verhaftetsein der Realität und der Alltagspraxis den eskapistischen Charakter unterstreichen, während er in (auto)biographischen Texten verdeckt bleiben kann. Die Eskamotierung des Hiatus von Fiktion und Historie ist kennzeichnend für das traditionelle lebensgeschichtliche Paradigma, das noch intensiv im Banne der Wissenschaftlichkeit steht und naturwissenschaftlichen Kriterien verhaftet zu sein scheint. Die Leser lebensgeschichtlicher Texte glauben sich in

2 Franz Grillparzer, Selbstbiographie. Residenz Verlag:Salzburg und Wien 1994. Vgl. etwa S. 61 und 164.

3 Die Hervorhebungen, gerade auch in den Zitaten, stammen, wenn nicht anders vermerkt, von der Verfasserin. 
mehrfacher Hinsicht abgesichert: Die Faktizität des empirisch gelebten Lebens wirkt, wie Siegfried Kracauer bereits 1930 luzide analysierte, als Garantie der Komposition ${ }^{4}$ und suggeriert die Ausblendung ästhetischer Verfahren. Im Gegensatz zum modernen Roman etwa gaukelt die traditionelle Biographik dem Leser ein überschaubares, beherrschbares Weltganzes vor, mit einem in sich konsistenten Subjekt im Zentrum, dessen Lebensgeschichte sinnvoll verläuft.

Hervorgerufen wird der Eindruck von Sinnhaftigkeit und Überschau infolgedessen zum einen durch das Festhalten an veralteten, weitverbreiteten Ich- und Weltvorstellungen, zum anderen durch den fast ausschließlichen Einsatz von Erzählmustern des 19. Jahrhunderts, die dem Leser durchaus vertraut sind und ihn in seiner vermeintlichen Lesekompetenz bestärken. Gerechtfertigt erscheint indes eine solche Darstellungsweise aus zwei Gründen: (Auto)biographisches Erzählen wird erstens als eine rein mimetische Handlung verstanden, als Wiedergabe - Nachschreiben - eines bereits gestalteten Geschehens, des Lebenslaufs. Zweitens ist eine konventionelle, ungebrochene, pragmatische Versprachlichung im nicht-fiktionalen Kontext üblich. Vom literaturwissenschaftlichen Standpunkt aus verwundert hingegen in erster Linie die Perpetuierung struktureller Anachronismen.

Auf der Folie der alternativen biographischen Paradigmen, wie sie sich seit Ende der sechziger Jahre in der deutschsprachigen Literatur zunehmend durchgesetzt haben, wird augenfällig, wie die Sinnkonstitutionsmechanismen des traditionellen Paradigmas funktionieren. Die Selbstbesinnung (auto)biographischen Erzählens, die Problematisierung seiner Kohärenz, die Aufdeckung seines Konstruktionscharakters und damit einhergehend eine Regenerierung biographischer Genres, verlief beispielhaft - über ästhetische Texte. Diese deckten die Essenzialität des retrospektiven Standpunkts in lebensgeschichtlichem Erzählen auf, was die traditionelle Biographik und Historiographie zugunsten der Prozeßhaftigkeit, d.h. der Vergegenwärtigung als Mimesis des Werdens (Droysen), und nach dem Prinzip der ästhetischen Harmonisierung der Gegensätze verdrängt hatte. Die Doppelstruktur bzw. Zweidimensionalität historiographischen und damit biographischen Erzählens ist von Roland Barthes, A.C. Danto und Hayden White konstatiert und untersucht worden. ${ }^{5}$ Rein sprachlich erzeugt wird die Mimesis des Bewegungsverlaufs durch die temporale Verfaßtheit des histori-

4 Siegfried Kracauer, Die Biographie als neubürgerliche Gattung. In: ders., Das Ornament und die Masse. Frankfurt/Main 1963. S. 75-80. S. 77.

5 Vgl. Roland Barthes, Historie und ihr Diskurs. In: Alternative, XI (1968), Heft 62/63. S. 171-180. S. 175.

Ders., Das semiologische Abenteuer. Frankfurt/Main 1988.

A.C.Danto, Erzählung, Erkenntnis und die Philosophie der Geschichte. In: Eberhard Lämmert, Erzählforschung. Stuttgart 1982. S. 643-659.

Hayden White, Metahistory. The historical imagination in nineteenth-century Europe. Baltimore/London 1973.

Ders., Die Bedeutung der Form. Erzählstrukturen in der Geschichtsschreibung. Frankfurt/Main 1990. 
schen/biographischen Diskurses: Durch die Satzfolge wird der Rückbezug zur Sprechsituation, der Behauptungssätze im allgemeinen charakterisiert, getilgt und eine sekundäre raumzeitliche Situierung ${ }^{6}$ geschaffen, was die Etablierung der Erzählung auf der Ebene des Énoncé ermöglicht. A. C. Danto radikalisiert Barthes' Einsichten: Er postuliert eine kognitive Asymmetrie, da sich der Erzähler logisch außerhalb der Ordnung der Ereignisse, die er beschreibt, befindet, denn er weiß, wie die Dinge zu Ende gingen, wie die Handlungen des Agenten im Lichte späterer Ereignisse zu beschreiben sind. ${ }^{7}$ Indes ist, vom kognitiven Standpunkt aus, die Offenheit der Zukunft in der Struktur der Erzählung für den Agenten eine Voraussetzung, weil nur dadurch die Möglichkeit der Zukunftsgestaltung, einer Wahl, gegeben ist. Danto resümiert vereinfachend: um Geschichten erzählen zu können, dürfen Dinge und Ereignisse nur wahrgenommen werden und beschrieben werden, wie sie historisch beschrieben werden können/.../von der Perspektive, die gewährt, daß die Ereignisse für sie /die Agenten/ in der Zukunft, aber für die Historiker in der Vergangenheit liegen. ${ }^{8}$

Neben diesem retrospektiven Vorgriff (Barthes), der im Erzählen zum Tragen kommt, genügt die Zielstrukturiertheit der Narration, um allein auf der Basis einer Aneinanderreihung disparater, relevanter Geschehnisse den Effekt eines Kausalnexus zu erzeugen. Die Chrono- logie, der Lebens-(ver)lauf, Temporalität und Prozessualität, zunächst gleichgültig ob in "Geschichtenform", d.h. narrativ, oder in Annalenform, konstituiert die wesentlichste Ordnungs- und Sinnbildungsachse. Aus der Erkenntnis, daß das biographische Erzählen /.../die Sachverhalte also von ihrem Resultat her ${ }^{9}$ strukturiert, wird eine konsequentielle Kategorie eingebracht, d.h. eine Sinnbildungskategorie, im Unterschied zur rein temporalen des Aussagemomentes. Es kommt bewußt/unbewußt zur Gleichschaltung zweier Kategorien, einer logischen und einer linguistischen:

Alles weist darauf hin, so Barthes in seiner Einführung, daß die treibende Kraft der narrativen Aktivität die Verwechslung von zeitlicher Folge und logischer Folgerung ist, das Nachfolgende in der Erzählung als verursacht von gelesen wird; die Erzählung wäre in diesem Fall die systematische Anwendung des in der Scholastik unter der Formel post hoc, ergo propter hoc angeprangerten logischen Irrtums, der durchaus der Wahlspruch des Schicksals sein könnte, dessen 'Sprache' die Erzählung im Grunde ist. $^{10}$

6 Wolf-Dieter Stempel, Erzählung, Beschreibung und der historische Diskurs. In: Reinhard Koselleck und Wolf-Dieter Stempel (Hg.), Geschichte - Ereignis und Erzählung. Poetik und Hermeneutik V. München 1973, S. 325-346. S. 327.

7 Danto, S. 654.

8 Ebda., S. 649.

9 Jochen Rehbein, Biographisches Erzählen. In: Lämmert, a.a.O. S. 51-73. S. 54.

10 Barthes, Das semiologische Abenteuer. S. 113. Vgl. auch S. 149. (Hervorhebung Roland Barthes) 
Besonders wirksam erweisen sich diese Verwechslungen zweier Kategorien in erzählten Lebensgeschichten, die als alltagsweltliche Deutungssysteme verstanden und unter dem Aspekt der Generierung von Orientierungswissen untersucht werden. Ausgegangen wird von dem Konzept, daß soziale Wirklichkeit hervorgebracht wird in Prozessen sinnhaften Handelns, ${ }^{11}$ wozu auch die mitlaufenden Interpretationsvorgänge von sich abwickelnden Handlungen und Ereignissen gehören. In diesem Sinne ist $\mathrm{Le}$ bensgeschichte /.../immer die von einem einzelnen Menschen erlebte und produzierte Lebensgeschichte. ${ }^{12}$ Was in Form von Lebensgeschichten oder -erzählungen sprachlich rekapituliert wird, ist folglich keine Abbildung der Realität sondern Interpretation. Insofern sind Lebensgeschichten zu verstehen als besondere Form der Erklärung, sie sind symbolische Konstruktionen, in denen Ereignisse und Handlungen interpretiert werden im Blick auf eine Sinnstruktur. ${ }^{13}$ Die darin produzierten Sinnbildungsprozesse können eine Intensität annehmen, daß der Lebenslauf eine ans Naturgesetzliche grenzende Folgerichtigkeit aufzuweisen scheint.

Infolge dieser Modellierung vom Ziel her scheint es angebracht, mit Fischer die Dynamik und strukturelle Offenheit lebensgeschichtlichen Erzählens zu betonen. Das bedeutet nicht nur, daß Lebensgeschichten abhängig von den Situationen, $Z$ wecken und Interaktionspartnern variierend konstruiert werden, sondern daß ihre Sinnstrukturen auch in der Zeitfolge modifiziert werden. Denn, die Orientierungsleistung und die Erhaltung lebensgeschichtlicher Konsistenz können nur erbracht werden, wenn vom prospektiven Fluchtpunkt aus die biographische Retrospektive als permanente Interpretation vorgenommen wird. ${ }^{14}$ Lebensgeschichtliche Integrations- und Orientierungsprozesse sind natürlich nur notwendig, wenn das Leben als Ansammlung kontingenter Daten erfahren wird. Aus Kontingenz wird mithilfe bestimmter invarianter Determinanten Konsistenz und Kohärenz konstruiert. Biographieren erfolgt in mündlicher Darstellung, aber auch im Großteil der schriftlichen, nach bestimmten Regeln, die Schütze für konversationelle Erzählungen feststellt. ${ }^{15}$ Das Leben oder Lebensfragmente müssen gerafft, zusammengefaßt geschildert werden, was Rehbein Prinzip der gebündelten Wiedergabe und Schütze Kondensierungszwang genannt haben. Die Selektion der Daten erfolgt immer unter bestimmten Relevanzaspekten, auf einen bestimmten Sinnhorizont zu. Zeitraffung gewährleistet zwar Übersichtlichkeit, bedeutet jedoch zugleich den Verlust von Vieldeutigkeit, Heterogenität, ist interpretierende Vereinfachung. Die zweite Regel, diejenige der Gestaltschließung, oder nach Jauß/Droysen

11 Wolfram Fischer, Struktur und Funktion erzählter Lebensgeschichten. In: Martin Kohli (Hg.), Soziologie des Lebenslaufs. Darmstadt und Neuwied 1978. S. 311-336. S. 311.

12 Werner Fuchs, Biographische Forschung. Opladen 1984. S. 95.

13 Fischer, S. 318.

14 Ebda., S. 319.

15 Fritz Schütze, Narrative Repräsentation kollektiver Schicksalsbetroffenheit. In: Lämmert, S. 568590. 
"Illusion des ersten Anfangs und definiten Endes"16 entspricht den Determinanten der Begrenzung nicht allein der aristotelischen Fabel, sondern definiert ebenso die Begriffe "Geschichte" (in der Bedeutung story nicht history) und "Ereignis". Gelingt die quasikausale Vernetzung nicht zur Gänze, so kann disparates Geschehen auch durch Detailakkumulation (nach Schütze Detaillierungszwang) verschleiert werden. Dementsprechend kann das (wissenschaftliche) Detail in der biographischen Belletristik ebenso zur Camouflage der Fiktionalität dienen, da die fiktionalen Einzelheiten vom faktischen Umfeld und durch die Einstellung des Lesers absorbiert werden. Es entsteht, um Barthes' gelungene Wortprägung in einer abgewandelten Bedeutung anzuwenden, ein Effekt des Realen. ${ }^{17}$

Sinnstiftend ist indes nicht nur die Kongruenz, sinnformend wirkt sich ebenso die Zeitkomponente aus. Die Komplexität, Simultaneität und Disparatheit der Biographieelemente, das, nach Fischer Sammelsurium dessen, was ein einzelner objektiv durchlebt hat, ${ }^{18}$ muß auf eine Abfolge reduziert werden. Aufgefädelt auf der Zeitachse - unabhängig davon ob streng chronologisch vorgegangen wird oder nicht, denn modelliert wird in jedem Fall vom Ende her - gewinnen die biographischen Daten an Stringenz, verloren gehen dabei zum Teil Heterogenität und Mehrdeutigkeit. Die Linearität wird in ihrem Sinnbildungseffekt unterstützt von fiktionalen, ästhetischen Verfahren: Bereits Droysen konstatierte für die Geschichtsschreibung das Wirken von Regeln, die in Analogie zur aristotelischen Bestimmung der Fabel zu sehen sind. Paul Ricoeur radikalisiert diese Position mit seiner These, daß wir unser Leben nicht erst im Nachhinein, sondern bereits in actu ästhetischen Strukturen nachmodellieren. ${ }^{19}$

\section{Vielfalt lebensgeschichtlichen Erzählens und das postmoderne Paradigma}

Vielfalt, breite Streuung in den verschiedensten Lebensbereichen sowie eine aus dem Funktionsangebot resultierende Relevanz charakterisieren lebensgeschichtliches Erzählen in allen Ausprägungen, vom anspruchsvollen literarischen Kunstwerk bis zu den massenhaft verschlungenen und emotional-sensationalistisch aufbereiteten "Lebensstories" der Boulevardblätter, von der Grabinschrift mit den Lebensdaten, der Minimal"biographie", bis zu dokumentstrotzenden, Genauigkeit vorexerzierenden

16 Hans Robert Jauß, Der Gebrauch der Fiktion in Formen der Anschauung und Darstellung der Geschichte. In: Reinhart Koselleck, Heinrich Lutz und Jörn Rüsen (Hg.), Formen der Geschichtschreibung. Theorie der Geschichte. Beiträge zur Historik Band 4, München 1982. S. 415-451.

17 Barthes, Historie, S. 180.

18 Fischer, S. 319.

19 Vgl. White, Bedeutung. S. 180. (Im Aufsatz: Die Metaphysik der Narrativität: Zeit und Symbol in Ricoeurs Geschichtsphilosophie. S. 175-193.) 
mehrbändigen Wälzern, von mündlichen Skizzen, zu Unterhaltungs- oder Forschungszwecken geliefert, bis hin zu jenen unter "Sachbuch" laufenden Verkaufsschlagern, die Lebensgeschichten in einer Mischung von Populärwissenschaft und Trivialliteratur präsentieren.

Allein in den Illustrierten und Wochenblätter nimmt Biographisches in verschiedenen traditionellen journalistischen Textsorten (Portraits, Nekrologe, Interviews, Leserbriefe in Beratungsecken, Dossiers mit thematischen Schwerpunkten, Aktuelles über Stars und Berühmtheiten, Kontaktanzeigen etc.), einen sehr hohen Prozentsatz ein. Ein Blick auf den Bildschirm genügt, um die Palette des (Auto)Biographischen mit dem Reichtum ihrer Farben und Töne in medienspezifischen Textsorten wahrzunehmen. In diesem Kontext stechen vor allem jene Sendungen ins Auge, die aufgrund eines seltsam obsoleten und doch verständlichen Authentizitätshungers der Zuschauer lebensgeschichtliche Fragmente - es handelt sich dabei fast ausschließlich um Lebensrisiken wegen Unfällen oder Verbrechen - in nachgespielten Versionen anbieten. ${ }^{20}$ Ganz besonders augenfällig ist bei dieser und ähnlichen Serien - etwa die Teilnahme an Polizeiaktionen - die Selbstverständlichkeit, mit der die Grenzen zwischen Faktischem und Fiktivem verwischt werden. Der Realitätseffekt des Fiktiven wird durch Kontamination mit dem Empirisch-Stattgefundenen erzeugt. Die Artifizialität des Nachgestellten wird ausgeblendet, angeboten wird es als "Veranschaulichung" von Geschehenem. Problematisch ist dabei nicht das Verfahren selbst, sondern das durch das Verwischen des Rahmenwechsels ausgelöste Spiel mit der Rezipientenwahrnehmung: Aus Wirklichkeitsdurst kann Wirklichkeitsverlust entstehen, wenn dem Zuseher die Wahrnehmungsperspektiven durcheinandergeraten und er die Fiktionsregeln eines Fernsehoder Filmgenres auf Geschehenes überträgt, wenn er erwartet, daß sich Wirklichkeit nach dramaturgischen Gesetzen strukturiert. Dies - der Einfluß der visuellen Medien auf die Wirklichkeitswahrnehmung - ist freilich ein weites, über diesen Beitrag hinausgehendes Feld.

Damit sind gleich zwei weitere Punkte angesprochen: ein drittes Paradigma sowie die Bedeutung ästhetischer und fiktionaler Verfahren für alltagsweltliche Textsorten. Das traditionelle und das alternative lebensgeschichtliche Paradigma müssen durch ein weiteres ergänzt werden, das man, ohne sich nun auf eine Begriffsdiskussion einlassen zu wollen, als das postmoderne Paradigma bezeichnen könnte. Während die Romanbiographie oder der historische Roman (in dessen Tradition es eher tendiert) möglichst "authentische" historische Darstellungen, das Vorspiegeln einer vergangenen Welt, d.h. die Fiktion als Realität anstreben, besteht in der Postmoderne dahingegen kein Bedarf mehr, Fiktion und Realität - wie noch in der Moderne - voneinander abzuheben. Wenn

20 Ich denke dabei an Serien über öffentliche Rettungsdienstleistungen, etwa Rettungstelephone, worin neben Ärzten und Krankenpflegepersonal auch noch die Feuerwehr und die Polizei eine zentrale Rolle spielen, und verschiedene spektakuläre Rettungsfälle von Schauspielern nachgestellt werden. Die tatsächlichen Opfer und Retter dürfen in eingeblendeten Interviews oder kommentierend dabei mitwirken. 
die Moderne eine Relativierung des Totalanspruchs von Kunst auf allen Niveaus war, dann betreibt die Postmoderne Integrations- und Vernetzungskunst. Die unendliche Reproduzierbarkeit des Wirklichen macht prinzipielle Differenzierungen obsolet, die Austauschbarkeit der Elemente wird jedoch durch die artistische Präzision in deren Platzzuweisung, sogar in ihren Konnotationen, verhindert. Der konstruktive Charakter, der in der Moderne deutlich herauspräpariert würde, geht in die Erzählung ein, wird narrativiert, nicht durch diskursive Thematisierung, sondern durch die Geschichten selbst. Die bewußt gesetzte und wahrgenommene Virtuosität in der Handhabung und Gegeneinandersetzung biographischer Muster produziert einen Überschuß an Sinn, der sich nicht in der Freude am Wiedererkennen vertrauter Muster konsumieren muß. Mit Vorliebe werden Mystifikationen der Autorschaft und der Textgenese miteingeflochten und mit einem Augenzwinkern aufrechterhalten: Die Frage nach der Echtheit wird mit dem Spiel des Fingierens von Authentizität substituiert. Die im weiteren typische Mehrfachcodierung manifestiert sich nicht in Kontaminationen durch andere Genres oder in hybriden Formen, wobei eine zentrale Textsorte von anderen modifiziert oder aufgeweicht wird, sondern der Text wird durch die Polyfunktionalität einzelner gleichwertiger Strukturelemente, die mehreren Genrecodes gleichzeitig angehören können, zu unterschiedlichen Genres vernetzt. Dadurch werden nicht nur verschiedene Leserkompetenzen angesprochen, d.h. die Texte können breitere und vielfältigere Leserkreise erreichen, sondern sie können auch von einem Leser nacheinander, abwechselnd oder zugleich auf den verschiedenen Netzebenen rezipiert werden. Die Auflösung der Genrehierachie und die bereits erwähnte Gleichschaltung der Bruchstücke oder Elemente beruht unter anderem auf der allgemeinen Dezentrierung und Parzellierung in der Kunst wie im Leben: Die Fragmente oder Textelemente fügen sich zu einer bruchlosen Oberfläche ohne Tiefe, zu einer glatten Haut ohne Risse. Es entsteht das, was Jameson eine neue Oberflächlichkeit ${ }^{21}$ nennt. Diese ist in den angeführten biographischen Texten mit großer sprachlicher und technischer Virtuosität sowie Subtilität gekoppelt. Hand in Hand mit dieser Vernetzbarkeit auf der Oberfläche geht der Verlust der Tiefe, der immer ein Verlust der Historizität ist. Jameson macht auf das Paradoxon einer nostalgischen Sehnsucht nach den Lebens- und Ausdrucksformen der Vergangenheit ${ }^{22}$ aufmerksam und der Unfähigkeit des "fragmentierten Subjekts", das in eine diffuse Räumlichkeit getaucht ist, sich auf der Zeitachse vorwärts und rückwärts zu bewegen: eine narzißtische Erstarrung gleichsam oder Lähmung durch das Spiegelbild. Eine authentische Historizität, wie sie zum Teil noch in der Moderne (und im alternativen Paradigma) möglich war, ist mit der postmodernen nostalgischen Kunstsprache laut Jameson schon deswegen inkompatibel, da sie eine Ästhetik vertritt, die vom Verschwinden des histori-

21 Fredric Jameson, Postmoderne - zur Logik der Kultur im Spätkapitalismus. In: Andreas Huyssen, Klaus R. Scherpe (Hg.), Postmoderne. Zeichen eines kulturellen Wandels. Reinbek bei Hamburg 1986. S. 45-102. S. 51, 54f.

22 Andreas Huyssen, Postmoderne - eine amerikanische Internationale? In: Huyssen/ Scherpe, S. 1344, S. 14. 
schen Referenten geprägt ist. Der historische Roman der Gegenwart kann es sich nicht mehr zur Aufgabe machen, die historische Vergangenheit einfach zu repräsentieren. Er 'repräsentiert' nur mehr unsere Vorstellungen und Stereotypen von dieser Vergangenheit. ${ }^{23}$ Was wir von einer historischen Persönlichkeit wissen, ist folglich ein ziemlich diffuses Megazeichen, das aus lauter Bildern besteht, und woraus lediglich ein Simulakrum (die Kopie einer Kopie, weil das Original verlorengegangen ist) entstehen kann. Da die Vergangenheit nur mehr in der Wiederholung (und nicht dem "Wieder-holen") greifbar ist, macht die Kunst der Imitate diese zu ihrem ästhetischen Prinzip: Eklektizismus, Epigonentum werden ins Positive umgemünzt. Die sich durch den Utopieverlust und den verlorenen Glauben an Aufklärung ausbreitende Resignation, Melancholie und Indifferenz soll mit dem Rekurs auf das Hedonistische und Ludistische wettgemacht werden. Betrieben wird ein zuweilen anmutig-heiteres, zuweilen ernsthaft- düsteres Spiel, worin sich der gespaltene Leser in verschiedenen Rezeptionsmodi gefallen kann.

Was hier zuletzt als eine lediglich der Literatur vorbehaltene Spielart lebensgeschichtlichen Erzählens erscheint, findet paradigmatische Parallelen und Entsprechungen in der Genreentwicklung mancher Medien, besonders des Films, der gerade in der virtuosen Handhabung der Genrepolyphonie und der Metafiktion eine Vorreiterrolle spielt, sowie in all den Bereichen des öffentlichen Lebens, wo zum einen mithilfe lebensgeschichtlicher Simulakren, den Images von Politikern etwa, Macht zu holen ist und zum anderen in publikumsgerechten Werbefiktionen der Prominenz den zu Voyeuren verkommenen Lesern und Zusehern ein Ersatzleben vorgespielt wird. Die Inszenierung des öffentlichen Lebens in den Medien - der Golfkrieg etwa ist zur Genüge analysiert worden - verweist auf die Tendenz zur allmählichen Vereinnahmung des Faktischen, Empirisch-Überprüfbaren durch literarische, ästhetische, fiktionale, narrative Muster und Verfahren. Bei Ricoeur heißt es, durchaus differenzierend und nicht manipulativ, menschliche Ereignisse als Produkte menschlicher Handlungen hätten die Struktur narrativer Texte. Ein sinnvolles Leben ist ein Leben, das mittels einer Fabel die Kohärenz einer Erzählung anstrebt. Historische Akteure präfigurieren ihr Leben als Erzählungen mit einem plot. ${ }^{24}$

\section{Erweiterung des Untersuchungsgebietes, Prototypisches in}

Textsorten und Genres der Lebensdarstellung

Die vorliegenden Überlegungen stellen eine äußerst komprimierte Fassung einiger wichtigerer Einsichten dar, die in einer umfassenden Abhandlung über theoretische Aspekte der biographischen Diskursivierung formuliert wurden; sie gingen von konkreten

24 White, Bedeutung. (Ricoeur) S. 180.(Hervorhebung Hayden White). 
literaturhistorischen und -wissenschaftlichen, nicht textlinguistischen Beobachtungen aus: von der literarischen Textproduktion, dem biographischen Boom in der deutschsprachigen Literatur der späten sechziger und siebziger Jahre, der, modifiziert, bis in die Gegenwart anhält, den beiden in dieser Zeit entstandenen Paradigmata - die hier bereits erörterten, nämlich das alternative und das postmoderne -, von einem anhaltenden Markterfolg (auto)biographischer Genres und Textsorten, die nach dem traditionellen Schema funktionieren, sowie von einem diesbezüglichen literaturwissenschaftlichen Defizit. ${ }^{25}$ Meinen Forschungen nach läßt sich das amöbe Phänomen "Biographie" indes nicht über eine auf literarische Texte beschränkte Untersuchung fassen, denn explizit literarische lebensgeschichtliche Genres - etwa die Biographie romancée oder biographische Theaterstücke - sind literaturtheoretisch weniger ergiebig, da die hierarchische Unterordnung der Referenzialität unter das dominant Poetische in einem ausgesprochen ästhetisch funktionierenden Text den Doppelcharakter, die Doppelgleisigkeit, der Rezeption verwischt. Diese wird evident in jenen Texten, die die Forschung im Grenzbereich zwischen Literatur und Wissenschaft angesiedelt hat; jedoch bringt das bisher zugrundegelegte antinomische, bipolare Schema keine einsinnige, überzeugende Antwort auf den beobachteten Widerspruch zwischen konventionellen Sinnstiftungs- und Integrierungsmodi der Diskursivierung und der Selbst- und Welterfahrung des Produzenten oder Rezipienten. Die Vergegenwärtigung der zahlreichen Genres und Textsorten lebensgeschichtlichen Darstellens machte deutlich, daß allgemeinere Aussagen gerade für nicht ausgesprochen literarische Genres lediglich auf einer funktionszentrierten, interdisziplinären und intertextuellen Basis gemacht werden können und diese wiederum Rückschlüsse auf Rezeption und Leserverhalten erlauben; neben der Berücksichtigung von Erkenntnissen der Erfahrungs- und Gesellschaftwissenschaften galt es, linguistisch-literarischen Wechselbeziehungen ebenso nachzugehen, wie es nun und in Zukunft ratsam ist bzw. sein wird, die Transformationen dieser Textsorten oder Genres nicht nur in den Printmedien zu verfolgen, sondern insbesondere Veränderungen in den akustischen und visuellen Kanälen in Betracht zu ziehen. Daraus ergab sich naturgemäß eine Erweiterung des Untersuchungsgebietes von den Bereichen der Wissenschaft und der Kunst auf die sehr weiten Felder der Alltagspraxis und der

Obwohl in der vorliegenden Arbeit das Autobiographische und Biographische unter den Sammelbegriff des "lebensgeschichtlichen Erzählens" subsumiert wird, weil es sowohl in der Funktionalität, den paradigmatischen Verknüpfungsstrategien sowie in der Rezeptionsart fundamentale Parallelen und Berührungspunkte gibt, muß hervorgehoben werden, da $B$ die autobiographische Vertextung in der (angloamerikanischen, deutschen und französischen) Forschung eine bevorzugte Position einnimmt; dieses gehobene Interesse spiegelt sich auch in der Forschung, die anregende und wichtige Ansätze hervorgebracht hat. Diese können jedoch weder plan auf die biographische Diskursivierung übertragen werden, da sie das Moment des Selbstbezuges in den Vordergrund schieben, noch enthalten sie Überlegungen, wie sie hier angerissen werden. - Die einzige rühmliche Ausnahme einer eingehenden, gewichtigen Studie in der Biographieforschung stellt das wichtige Buch (mitsamt einigen Aufsätzen) Helmut Scheuers Biographie. Studien zur Funktion und zum Wandel einer literarischen Gattung vom 18. Jahrhundert bis zur Gegenwart (Stuttgart 1979) dar, das literaturhistorisch, philosophie- und sozialgeschichtlich orientiert ist. 
Freizeitgestaltung; ob der hier gewählte Sammelbegriff des lebensgeschichtlichen Erzählens oder Darstellens eher eine Diskursart bezeichnet oder nach Heinemann/ Viehweger eher einen Texttyp, ${ }^{26}$ muß einstweilen noch offen bleiben. Siegfried J. Schmidt plädiert für das von Fishelov vorgeschlagene Wittgensteinsche Konzept der Familienähnlichkeit, das mir äußerst tauglich erscheint, da es in synchroner Sicht ergänzt wird von Intertextualität und diachron von sogenannter common ancestry, das entscheidende Moment von Stabilität in der Beziehung zwischen den in vielen anderen Aspekten voneinander unterschiedlichen Mitgliedern einer Gattungsfamilie. ${ }^{27}$

Die doppelte Orientierung auf die Funktionalität - auf die individuellen und sozialen Funktionen, die der Texttyp/die Diskursart lebensgeschichtlichen Darstellens oder Erzählens erfüllt, sowie auf die Art und Weise, wie die verschiedenen Textsorten und Genres funktionieren - eröffnet, wie Siegfried J. Schmidt ausführt, nicht nur einen großen Reichtum an Unterscheidungsaspekten, sondern folgt den bisher entwickelten erkenntnistheoretischen Hypothesen, wonach Eigenschaften und Bedeutungen nicht in den Objekten, Ereignissen und Medienangeboten selbst liegen, sondern ihnen von Aktanten in Kognitionen und Kommunikationen mit Hilfe von Unterscheidungen und Benennungen zugeschrieben werden. ${ }^{28} \mathrm{Zu}$ dieser Erkenntnis gelangte ich im Laufe meiner Untersuchungen, noch ohne auf entsprechende textlinguistische oder konstruktivistisch-systemtheoretische Ansätze zu Textsorten und Gattungen zu rekurrieren; dies scheint mir ein weiterer Beweis für die Relevanz und Tauglichkeit eines funktionalen, kommunikativen und handlungsbestimmten Zugangs zu sein. Den zweiten Befund, die These von der Interdependenz und gegenseitigen Beeinflussung verschiedener Textsorten und Genres mit einer gemeinsamen Funktion - hier der "lebensdarstellenden", (auto)biographischen -, fand ich hingegen in der Forschung lediglich angedeutet. Wiewohl die Klassifikationsvorschläge von Wolfgang Heinemann und Dieter Viehweger, Matthias Dimter und Siegfried Heusinger ${ }^{29}$ ebenso wie die Überlegungen

26 Wolfgang Heinemann/ Dieter Viehweger, Textlinguistik. Eine Einführung. Tübingen 1991. S. $138 / 139$.

27 Siegfried J. Schmidt, Kognitive Autonomie und soziale Orientierung. Konstruktivistische Bemerkungen zum Zusammenhang von Kognition, Kommunikation, Medien und Kultur. Frankfurt/Main 1994. S. 182, vgl. auch S. 181 und 183.

28 Ebda., S. 165/166.

29 Heinemann/Viehweger, a.a.O.

Matthias Dimter, Textklassenkonzepte heutiger Alltagssprache. Kommunikationssituation, Textfunktion und Textinhalt als Kategorien alltagssprachlicher Textklassifikation. Tübingen 1981.

Siegfried Heusinger, Pragmalinguistik. Texterzeugung, Textanalyse; Stilgestaltung und Stilwirkung in der sprachlichen Kommunikation. Ein Lehr- und Übungsbuch. Frankfurt/Main 1995. Vgl. besonders auch Beispiel "Lebenslauf" im Beitrag desselben Autors des vorliegenden Sammelbandes.

Vgl. auch Christiane Nord, Textanalyse und Übersetzen. Heidelberg 1991.

Robert-Alain de Beaugrande und Wolfgang Ulrich Dressler, Einfïhrung in die Textlinguistik. Tübingen 1981. S. 188-193. 
von Siegfried J. Schmidt und Gebhard Rusch ${ }^{30}$ zwar eine Reihe von Anregungen zur Strukturierung und Eruierung von Unterscheidungsmerkmalen boten, entsprach keines der Modelle der Spezifik meines Untersuchungsgegenstandes zur Gänze, am geeignetsten jedoch erschien mir die Mehrebenenklassifikation von Heinemann/Viehweger, weil sie im Gegensatz zu den meisten - auch der aufschlußreichen und stimulierenden von Rusch ${ }^{31}$ - nicht primär über Merkmale operiert und dabei die in meinem Kontext wesentliche syntagmatische Verknüpfungsebene zu akzentuieren erlaubt. Diese wird etwas modifiziert als Basis uibernommen, wobei die Aufmerksamkeit zum einen auf Forschungsdefizite und zum anderen auf das Protoptypische "lebensdarstellender" Texte gelenkt werden soll:

\section{Voraussetzungen}

1. Die Diskursart oder der Texttyp "lebensgeschichtliches Erzählen" oder "Lebensdarstellung" ist definiert durch

a. das Thema - "Leben"

b. durch die Diskursivierungs/Verbalisierungsart "Erzählen" oder "Darstellen"

c. Da die grundlegende Aporie nach Barthes darin besteht, daß es keine Deckung zwischen der mehrdimensionalen Ordnung des Wirklichen und der eindimiensionalen der Rede gibt, bedeutet Verbalisierung zugleich Produktion und Anwendung gesellschaftlich sanktionierter Sinnstiftungs- und Ordnungsprinzipien. ${ }^{32}$

2. Aus dem gerade Angeführten geht hervor, daß lebensgeschichtliches Erzählen/Darstellen auf Komplexitätsreduzierungen beruht:

a. dem Ignorieren der oben angeführten Aporie - Metasprachliches, Überlegungen zum Code, Kontext und Kanal werden vor allem in ästhetischen Texten geboten;

b. der selbstverständlichen Handhabung der lenbensdarstellenden Funktionsvielfalt (s. unten).

(c. Komplexitätsreduktionen als prototypische Merkmale des Texttyps wirken durch die Bevorzugung unreflektiert eingesetzter mimetischer Vertextungsstrategien häufig anachronistisch.)

$30 \quad$ Vgl. Schmidt, a.a.O. S. 199.

31 Vgl. Ebda., S. 178: Als solche Eigenschaften bestimmt Rusch wahrnehmungsmäßige, kommunikationsbezogene und nutzungsbezogene Eigenschaften. Ebenso S. $189 \mathrm{f}$.

32 Roland Barthes, Leçon/Lektion. Antrittsvorlesung im Collège de France. Frankfurt/Main 1980. S. 33: Man könnte sich eine Geschichte der Literatur vorstellen oder besser: der Hervorbringung der Rede, die die Geschichte der - oft ganz aberwitzigen - verbalen Notbehelfe wäre, die die Menschen benutzt haben, um das zu reduzieren, zu zähmen /..., was immer ein Delirium ist, nämlich die fundamentale Nicht-Adäquatheit von Rede und Wirklichem. 


\section{FUNKTIONSTYPEN:}

1. Keines der berücksichtigten Klassifikationsmodelle führt globale interaktive Funktionen (d.h. der Interaktion Gesellschaft - Individuum) trotz ihrer expliziten Handlungsorientierung an. (Rusch gliedert - meiner Meinung nach einleuchtend - diese Funktionen den sogenannten properties of use, der Nutzung zu. ${ }^{33}$ ) $\mathrm{Da}$ sie für lebensgeschichtliches Erzählen oder Darstellen von fundamentaler Bedeutung sind, werden sie hier an erster Stelle angeführt:

1.1 Sozial- und individualpsychologische Konstruktionen: Identitäts- und Gruppenbewußtsein

\subsubsection{Gruppen/Gesellschaftsspezifisch: gruppen/gesellschaftskonstituierend}

(Zugehörigkeit zu einer Gruppe/Klasse/Schicht usw. wird durch lebensgeschichtliches Darstellen und seine Identfikationsangebote geleistet - etwa Bürgertum, Frauenbewegung, Emigranten...)

\subsubsection{Individuell: Sozialisation und Individuation}

(Vergleich mit anderen, Identifikation und Absetzung, identitätsstiftende Konstruktionsstrategien)

1.2 Dominierende Interaktionsbereiche:

\subsubsection{Informationsvermittlung - Wissenserwerb}

(breites Spektrum: vgl. 2.4)

1.2.2 Informationsspeicherung - kollektives Gedächtnis (Hagiographie, Historiographie: Hier ist die Differenzierung in gesellschaftlich sanktionierte Erinnerung und subversive Gedenk- und Gedächtnisformen sehr wichtig, die jedoch vor allem von ästhetischen Texten geleistet werden - vgl. 1.2.4.1.)

Dazu gehören lebensdarstellende publizistische Textsorten, Nekrologe in allen Medien (auch Lobgesänge und Grabreden), natürlich Biographien mit wissenschaftlichem Anspruch etc.

\subsubsection{Informationsverarbeitung - Normierung - Steuerung}

(ethisch-didaktisch: Viten, Legenden, exemplarische Biographien etc.)

1.2.4 Kompensation-Regenerierung: Bereiche/Funktionen, die von den anderen Sozialsystemen nicht gedeckt werden (Situationstyp: Freizeit - Unterhaltung)

1.2.4.1 Schaffung von Freiräumen und Alternativmodellen - Dynamisierung, Erhaltung der Flexibilität und Kreativität, Offenheit etc. (Situationstyp: Kunst)

1.2.4.2 Problemlösungsmodelle: Lebenshilfe (Erfahrungsbücher, Sachbücher, Beratungsecken, Funkberatung, Kontaktsendungen...)

\subsubsection{Konsolation, Ablenkung}


(biographische Trivialliteratur und -filme, Regenbogenpresse mit Interviews, Portraits, TV-Serien mit Aktuellem über Stars und Prominente...)

2. Nach dem Kommunikationsmodell und den sprachlichen Funktionen, wie sie Jakobson entwickelt hat:

2.1 Senderseite: selbstdarstellend (Heinemann/Viehweger) oder emotiv, expressiv

Textsorten mit dieser Dominanz: therapeutisches Gespräch, Briefe(bzw. Teile von Briefen), Tagebuch(eintragungen), konversationelle Erzählungen, Vorstellungsgespräche etc.

2.2 Code: metasprachlich (diese Funktion fehlt bei Heinemann/Viehweger)

Textsorten mit dieser Dominanz: (Auto)biographien des alternativen Paradigmas

2.3 Kanal: phatisch, kommunikativ (Heinemann/Viehweger)

2.3.1 Textsorten mit dieser Dominanz: vor allem konversationelle Gespräche (verschiedene typische Situationen: etwa Transportmittel, Krankenhaus, gesellschaftliche Anlässe)

2.3.2 Typen: verbal - non verbal (Photos, Filme etc.)

(Kombination: Ausstellungen, Kataloge etc.)

2.3.3 mündlich - schriftlich - visuell

Beispiele: Gespräche verschiedener Art, lebensdarstellende Funktexttypen (Interview, Feature) - vom Lebenslauf bis zur wissenschaftlichen Biographie in mehreren Bänden - Videos, Filme;

2.4 Kontext: referentiell, informativ (Heinemann/Viehweger)

Diese sprachliche Funktion dominiert zwar nicht in allen lebensgeschichtlichen Erzählungen, ist jedoch intensiv präsent, deshalb könnten ausnahmslos alle Textsorten und Genres angeführt werden.

\subsection{Botschaft/Nachricht: poetisch}

Die Bezeichnung "ästhetisches Wirken" von Heinemann/Viehweger vereinigt alle vier dort angegebenen sprachlichen Funktionen; dieser Vorschlag überzeugt wegen der Funktionsvielfalt lebensgeschichtlichen Erzählens, jedoch erscheint mangelhaft, daß das Ästhetische lediglich mit der Markierung "fiktive Welt" versehen wird, ohne Hinweis auf die Polyfunktionalität und Polyvalenz sowie Autoreferentialität ästetischer Texte.

Genres mit dieser Dominanz: "literarische (Auto)biographien", (auto)biographische Erzählungen, Romane, Filme, Hörtexte...

2.6 Empfängerseite: konativ, steuernd (Heinemann/Viehweger)

Die Rezeptionsseite ist, wie bereits aus den interaktiven Funktionen deutlich geworden ist, besonders wichtig, denn lebensgeschichtliche Erzähl- oder Darstellungstexte wollen stärker als andere Textsorten eine Wirkung - Handlungen, Reaktionen - von seiten des Rezipienten erzielen; charakteristisch dafür sind in der Frühzeit Viten und Legenden mit 
einer ethisch-didaktischen Funktion, ganz bedeutend sind alltagsweltliche Lebensdarstellungen, etwa Lebensläufe, in Empfehlungsschreiben oder bei Bewerbungen; die Werbung operiert gleichfalls damit - mit Lebensgeschichten für Produkte oder für Personen (Politiker etc.). - Auf die spezifisch doppelgleisige Rezeption geht der Schlußabsatz ein.

3. Funktionsvielfalt (unter 1. und 2.) ist für den Großteil lebensdarstellender Textsorten und Genres prototypisch.

\section{SITUATIONSTYPEN: (nach Heinemann/Viehweger)}

1. Interaktionale Rahmentypen: kommunikative - Konversation, Brief; nicht kommunikativ: praktisch - Lebenslauf bei Bewerbungen, Werbung mit Lebensgeschichten; geistig-theoretisch - nach Medien unterschieden etc.

2. Soziale Organisation - Tätigkeitsbereiche: weniger institutionalisiert: Freizeit - Literatur, TV, Rundfunk, Zeitungen; institutionalisiert: Wissenschaft, Religion, Kultur

3. Anzahl der Partner in der Situation (prototypisch für einzelne Textsorten, nicht für den Texttyp)

4. Dominanz oder Symmetrie der Kommunikationspartner:

dominant asymmetrisch (Ausnahme: Kontaktsendungen, psychoanalytisches Gespräch...)

prototypisch: Unterscheidung Autobiographie - Biographie $\rightarrow$ in der Autobiographie bürgt der Autor/Aussagesubjekt und Aussageobjekt für die "Authentizität" der Aussage ("Vertrag" zwischen Autor und Leser)

5. Grundtypen der Umgebungssituation, vgl. 2.3 (Kanal)

Es fehlt die für Lebensdarstellungen sehr wesentliche Unterscheidung in die offizielle und private Kommunikationssphäre (nach Heusinger "Kommunikationsbereich").

\section{VERFAHRENSTYPEN}

Prototypisch: Bedeutung der syntagmatischen Textbildungsschemata (vgl. Abschnitt I) 1. Dominant narrativ (auch deskriptiv, argumentativ, reflexiv...), d.h. Konstruktion einer Ereignisstruktur (Gestaltschließung, d.h. Anfang-Ende, Kondensierung, Detaillierung)

Ausnahme: stichwortartiger Lebenslauf in Annalenstruktur

2. Retrospektives Erzählen - Zielorientierung 
3. Vernüpfungsstrategien: Chrono-Logie, konsekutive Kategorie wird zur konsequentiellen $\rightarrow$ Sinnstiftung

4. Effekt des Realen: - Vergegenwärtigung als Mimesis des Werdens

- Detailakkumulation

\section{TEXTSTRUKTURIERUNGSTYPEN}

1. Textsortenspezifisch, paradigmenabhängig:

restriktiv: Lebenslauf, Nekrolog, Kontaktanzeige etc.

2. Gliederungs/Sequenzierungsprototypik:

makrostrukturell: - chronologisch

- nach Lebensphasen, Höhepunkten im Leben oder Schaffen

- nach Deutungsmustern (psychoanalytisch, soziologisch...)

(Nach der Referenzialität der Makro- oder der Mikrostruktur lassen sich fiktionale und nicht fiktionale Texte unterscheiden.)

3. Längen/Umfangabhängig

restriktiv: z.B. bio- und bibliographische Angaben zur Person (Vorstellung von Fachkollegen oder in Publikationen)

\section{FORMULIERUNGSMUSTER}

Besonders bei restriktiven alltagsweltlichen Textsorten, berühmt auch sog. "Geburtssatz".

Lebensgeschichtliches Erzählen und Darstellen erfüllt - das dürfte aus meinen Ausführungen zur Genüge deutlich geworden sein - eine Reihe bedeutender sozialer und kommunikativer Funktionen; doch einen Teil seiner ungebrochenen Aktualität und seines Reizes verdankt es weniger ehrenwerten menschlichen Bedürfnissen und Impulsen: Es schöpft auch, freilich nicht allein und nicht primär, aus Indiskretion, Sensationslüsternheit und Erfahrungshunger. Seine Anziehung wird jedoch letztlich - auch das ist meines Erachtens nach für die meisten seiner Genres und Textsorten prototypisch - aus einem spezifischen widersprüchlichen Rezeptionsvorgang generiert, der der ambivalenten Struktur des Genres und der Leserbedürfnisse entspricht. Die der Sprache inhärente Funktionsvielfalt kann bei verschiedenen Textsorten zugleich, nacheinander oder hierarchisch gestaffelt aufgenommen werden, was ein doppel- bzw. mehrgleisiges Lesen zeitigt. (Auto)biographische Texte - gleichgültig ob fiktional, faktisch oder pragmatisch - werden zunächst vorrational und affektiv aufgenommen, in ihrem "Re- 
alitätsgehalt" geglaubt. Damit dienen sie vorbewußter (und affektiver?) Selbst- und Weltvergewisserung, auch über die Beherrschbarkeit der Objekte über Sprache oder der Beherrschung der Sprache anstelle der Objekte. Dieser Rezeptionsmodus produziert Lustgewinn entweder in der Bestätigung durch Wiederholung des Bekannten oder in der Triebbefriedigung (der Neugier). Ins Bewußtsein gehoben, wird der Identifikationscharakter der Rezeption negiert, der Text - falls das die Situation erfordert, der Leser dazu ermuntert wird oder selbst dazu neigt - in seinem Konstruktionscharakter, seiner Zeichenhaftigkeit, seiner Fiktionalität oder Fingiertheit, wahrgenommen. Dieser zweite Rezeptionsmodus ist dominant rational, bewußt reflexiv und regulierend. In Anschluß an das doppelschichtige Verhalten, das Lotman beim Spiel diagnostiziert, wird die These aufgestellt, die Rezeption vor allem biographischer Texte sei markiert durch das simultane Auftreten der beiden Rezeptionsmodi sowie durch ein ständiges $O s$ zillieren zwischen diesen beiden oder auch noch weiteren. Meine These versteht sich nicht primär als Versuch, die postmoderne Befindlichkeit in ihrer Aufweichung der Gegensätze und der Gleichzeitigkeit von Widersprüchlichem in den Diskurs über Textsorten und Genres einzubringen, sondern sieht sich in erster Linie als Plädoyer für dynamischere, flexiblere Modelle, die auch Textsorten und Genres von hoher Komplexität zu fassen imstande wären und damit einen wichtigen Beitrag zur Literaturwissenschaft, nicht nur zur Linguistik, leisten könnten.

Povzetek

PARADIGME IN BESEDILNE VRSTE ŽIVLJENJEPISNEGA PRIPOVEDOVANJA

Na podlagi literarnozgodovinskih izsledkov v nemški književnosti zadnjih trideset let razvija razprava tri biografske paradigme: tradicionalno, alternativno in postmodernistično. Prvo, ki je postala razvidna prav iz njene tematizacije biografiranja $v$ alternativni paradigmi, označuje povzemanje pripovednih in miselnih vzorcev o celostnem subjektu, kot jih pozna 19. stoletje in ki v sodobnem času delujejo precej anahronistično. Da bi lahko odgovorili prav na vprašanje o trajnem uspehu biografskih besedil, ki ga estetsko obeležena dela zaradi dominantnosti poetične funkcije ne razkrivajo $v$ zadostni meri, je potrebno področje raziskave razširiti iz literarnega in znanstvenega področja predvsem na pregled številnih pragmatičnih življenjepisnih besedilnih zvrsti in njihovih funkcij.

(Avto)biografsko pripovedovanje kot skupni termin za besedilne zvrsti in vrste $z$ določenimi pomembnimi socialnimi oz. socializacijskimi in individualnimi funkcijami deluje predvsem sintagmatično in ima naslednje značilnosti: V jeziku povzema procesualnost, ne glede na to, da pripovedovalec govori iz retrospektive. To povzroča identifikacijo dveh različnih kategorij, časovne in vzročne (kronologija). Tako (avto)biografiranje vnaša $\mathrm{v}$ kontingenco življenja urejevalno dimenzijo osmišljanja, saj pripovedi sintetizira v dogodkovno strukturo.

$\mathrm{V}$ tretjem delu se razprava ukvarja $\mathrm{z}$ možnostmi in problemi lingvistične klasifikacije življenjepisnih pripovednih besedilnih zvrsti in vrst ter ponazarja na večplastnem modelu tudi pomankljivosti obstoječih razčlemb. Čar (avto)biografskega pripovedovanja izhaja po mnenju avtorice ne le iz številnih funkcij, ki jih tovrstna besedila izpolnjujejo, temveč predvsem iz posebne recepcije: Ta namreč niha med različnimi dominantnimi jezikovnimi funkcijami ali jih lahko celo sočasno aktualizira. 